\title{
Dérivations et Idéaux Réels Invariants
}

\author{
par \\ Herwig HAuSER* et Jean-Jacques RisLeR**
}

\section{Introduction}

Seidenberg a initié dans $[\mathrm{S}]$ l'étude systématique des idéaux de fonctions qui sont invariants par les dérivations. Le concept d'algèbre tangente d'une variété affine (ou d'un idéal)-collection des champs dans l'espace ambiant qui sont tangents à la variété (ou dérivations qui préservent l'idéal), aussi appelés champs logarithmiques à cause de la dualité avec les formes-constitue alors le lien entre la géométrie et la théorie des algèbres de Lie [H-M].

L'étude de $[\mathrm{H}-\mathrm{M}]$ est géométrique: du point de vue algébrique, elle ne considère que des idéaux réduits, le corps de base étant algébriquement clos. Dans le cas des réels, le concept d'idéal réduit n'a plus de signification géométrique. En essayant de généraliser les résultats de $[\mathrm{H}-\mathrm{M}]$ au cas réel, il est ainsi apparu qu'il était naturel de considérer aussi le cas des idéaux non réduits. Cet article commence donc par l'étude des relations entre l'algèbre tangente d'un idéal $I$, et l'algèbre tangente de sa racine $\sqrt{I}$, valable sur un corps arbitraire. En excluant les idéaux avec composantes immergées, nous montrons que les algèbres tangentes de $I$ et $\sqrt{I}$ coïncident si et seulement si $I$ est l'intersection de puissances symboliques de ses idéaux premiers minimaux associés. En présence de composantes immergées la situation n'est plus controllable comme le montrent les exemples les plus simples.

Nous abordons ensuite le cas de la géométrie réelle. Les résultats sont énoncés dans le cadre des germes de fonctions analytiques, mais sont aussi valables dans le cas algébrique, pour des idéaux d'un anneau de polynômes sur $\mathbf{R}$, ou d'un localisé d'un tel anneau. Nous montrons que chaque dérivation qui laisse invariant un idéal dans l'anneau des séries convergentes stabilise aussi sa racine réelle (concept qui est l'analogue dans le cas réel de la racine dans le cas complexe). De plus, les algèbres tangentes d'un idéal réduit et de sa racine

Communique par K. Saito, le 26, octobre, 1998.

1991 Mathematics Subject Classification(s): 32B10, 17B66, 14B05

* Mathematisches Institut, Universität Innsbruck, A-6020 Autriche

** Institut de Mathématiques, Université Paris 6, F-75252 France 
réelle coïncident si et seulement si les deux idéaux coïncident, i.e., si l'idéal de départ est réel.

Nous généralisons enfin au cas réel le résultat essentiel de $[\mathrm{H}-\mathrm{M}]$, à savoir que dans le cas algébrique affine, l'algèbre des champs tangents à une variété (plongée dans $\mathbb{R}^{n}$ ) caractérise cette variété. Le même résultat est vrai pour les germes analytiques dans $\mathbb{R}^{n}$, à condition que leur dimension soit $\geq 3$. Nous en donnons deux démonstrations; la première est basée sur la description des algèbres tangentes d'une variété réelle par des propriétés d'algèbres de Lie, la deuxième utilise l'équivalence entre les variétés complexes et leurs algèbres tangentes établie dans $[\mathrm{H}-\mathrm{M}]$ pour en déduire par passage à la partie réelle le résultat correspondant réel.

Nous remercions le referee pour la lecture attentive et des suggestions utiles. Cette collaboration a été supportée par un programme d'échanges scientifiques Franco-Autrichien (action intégrée Franco-Autrichienne "Amadeus").

\section{Dérivations et Idéaux Non Réduits}

On note $\mathcal{O}$, respectivement $\mathcal{O}^{\mathbf{C}}$, l'anneau des germes de fonctions analytiques réelles, resp. complexes, à l'origine de $\mathbf{R}^{n}$, resp. $\mathbb{C}^{n}$. L'anneau $\mathcal{O}^{\mathbf{C}}$ s'identifie naturellement au produit tensoriel $\mathcal{O} \otimes_{\mathbf{R}} \mathbb{C}$.

Nous allons dans ce paragraphe donner quelques définitions et propriétés élémentaires des dérivations laissant stable un idéal $I$, et considérer le cas où $I$ n'est pas réduit.

Soit $\mathbb{D}$ l'algèbre de Lie des dérivations de $\mathcal{O}$, et $\mathbb{D}^{\mathrm{C}}$ celle de $\mathcal{O}^{\mathrm{C}}$. Si $I$ est un idéal de $\mathcal{O}$, nous noterons $\mathbb{D}_{I}$ le $\mathcal{O}$-sous-module de $\mathbb{D}$ formé des $D \in \mathbb{D}$ telles que $D(I) \subset I$. Il est clair que $\mathbb{D}_{I}$ est aussi une sous-algèbre de Lie de $\mathrm{D}$. Dans tout ce paragraphe, les résultats formulés pour $\mathcal{O}$ s'appliquent aussi à $\mathcal{O}^{\mathrm{C}}$.

Le lemme suivant est pour l'essentiel contenu dans $[\mathrm{S}]$. Ce lemme, énoncé pour l'anneau $\mathcal{O}$, est valable pour tout $k$-algèbre Noethérienne, $k$ étant un corps. Pour $\wp$ un idéal premier de $\mathcal{O}$, on désigne par $\mathcal{O}_{\wp}$ le localisé de $\mathcal{O}$ en $\wp$.

Lemme 1. (a) Pour $I$ un idéal de $\mathcal{O}$, on a $\mathbb{D}_{I} \subset \mathbb{D}_{\sqrt{I}}$.

(b) Pour $I, J \subset \mathcal{O}$ on a $\mathbb{D}_{I} \cap \mathbb{D}_{J} \subset \mathbb{D}_{I \cap J}$.

(c) Pour $I \subset \mathcal{O}$ et $\sqrt{I}=\wp_{1} \cap \cdots \cap \wp_{s}$, posons $I_{i}=I \mathcal{O}_{\wp_{l}} \cap \mathcal{O}$. Alors $\mathbb{D}_{I} \subset$ $\mathbb{D}_{I_{1}} \cap \cdots \cap \mathbb{D}_{I_{s}}$.

(d) Si I n'a pas de composantes immergées, $\mathbb{D}_{I}=\mathbb{D}_{I_{1}} \cap \cdots \cap \mathbb{D}_{I_{s}}$.

Démonstration. Soient $f \in \sqrt{I}$ et $D \in \mathbb{D}_{I}$. Il existe un entier $p$ tel que $f^{p} \in I$, d'où $D f^{p}=p f^{p-1} D f \in I$. Appliquant $p$ fois $D$, on obtient un élément $g$ de $A$ tel que $(D f)^{p}+f g \in I$, d'où $D f \in \sqrt{I}$. Ceci prouve (a), et (b) est évident. Démontrons (c). Soit $D \in \mathbb{D}_{I}$, et $f \in I_{l}$. Il existe $a \in \mathcal{O} \backslash \wp_{l}$, tel que af $\in I$, par définition de $I_{i}$. Soit $D \in \mathbb{D}_{I}$. Alors $D\left(a^{2} f\right)$ et donc af $D a+a^{2} D f$ 
appartiennent à $I$. On en déduit que $a^{2} D f \in I$, d'où $D f \in I_{l}$, ce qui montre $D \in \mathbb{D}_{I_{i}}$. Enfin, (d) résulte de (b) et (c), car si $I$ n'a pas de composantes immergées, on a $I=I_{1} \cap \cdots \cap I_{s}$ par définition de la décomposition primaire.

Etudions maintenant dans quel cas on peut avoir l'égalité $\mathbb{D}_{I}=\mathbb{D}_{\sqrt{I}}$.

Lemma 2. Soient $m$ l'idéal maximal de $\mathcal{O}$ et I un idéal propre de $\mathcal{O}$. Les conditions suivantes sont équivalentes.

(a) $\mathbb{D}_{m}=\mathbb{D}_{I}$.

(b) $\mathbb{D}_{m} \subset \mathbb{D}_{I}$.

(c) $I=m^{k}$ pour un entier $k>0$.

Démonstration. Les implications $(\mathrm{a}) \Rightarrow(\mathrm{b})$, et $(\mathrm{c}) \Rightarrow(\mathrm{a})$ sont immédiates. Montrons que (b) implique (c). On choisit un ordre total sur $\mathbf{N}^{n}$, noté " $\leq$ ", qui est compatible avec l'addition de $\mathbf{N}^{n}$ et le degré total. C'est-à-dire que $\alpha \leq \beta$ implique $\alpha+\gamma \leq \beta+\gamma$ pour tout $\gamma \in \mathbf{N}^{n}$ et $|\alpha| \leq|\beta|$, où $|\alpha|$ est la somme des composantes de $\alpha$. Soit $x_{1}, \ldots, x_{n}$ un système régulier de paramètres de $\mathcal{O}$ de sorte que $\mathcal{O}=\mathbf{R}\left\{x_{1}, \ldots, x_{n}\right\}$. Notons in $f=x^{\alpha}$ le monôme initial de $f$. C'est le monôme de plus petit exposant (pour l'ordre $\leq$ ) dans le développement en série de $f$. Pour un idéal $I$ de $\mathcal{O}$, in $I$ sera l'idéal initial de $I$ engendré par tous les in $f$ pour $f \in I$. Fixons $i, j$ et soit $D \in \mathbb{D}_{m}$ la dérivation $D=x_{i} \partial_{\mathrm{x}_{\mathrm{j}}}$. On a alors

$$
D x^{\alpha}=\left\{\begin{array}{lll}
0 & \text { si } & \alpha_{\jmath}=0, \\
\alpha_{J} x^{\alpha-e_{\jmath}+e_{l}} & \text { si } & \alpha_{\jmath} \neq 0,
\end{array}\right.
$$

en notant $e_{l}$ les éléments de la base canonique de $\mathbf{R}^{n}$. Si $f \in I$ et in $f=x^{\alpha}$, choisissons un indice $j$ tel que $\alpha_{J} \neq 0$. Alors à cause de $(b)$ on a in $(D f)=$ $\frac{1}{\alpha_{J}} D($ in $f)=x^{\alpha+e_{1}-e_{l}} \in$ in $I$. Ceci entraîne que pour tout monôme $x^{\alpha} \in$ in $I$, on a aussi $x^{\alpha+e_{1}-e_{J}} \in$ in $I$. Soit maintenant $k>0$ minimal tel que $I \subset m^{k}$, et soit $f$ un élément de $I$ d'ordre $k$. Alors in $f=x^{\alpha}$ satisfait $|\alpha|=k$ puisque $\leq$ est compatible avec le degré total. On déduit de ce qui précède que tous les monômes de degré $k$ appartiennent à in $I$, donc $m^{k} \subset$ in $I \subset$ in $m^{k}=m^{k}$ d'où in $I=$ in $m^{k}$.

Montrons que cette égalité et l'inclusion $I \subset m^{k}$ impliquent $I=m^{k}$. Divisons un élément $g$ de $m^{k}$ par l'idéal $I$ selon le théorème de division de Grauert-Hironaka, cf. [H2], Theorem 1, (6), p. 261. Alors $g \equiv h$ modulo $I$, où $h$ appartient à un sous-espace vectoriel $\Delta(I)$ de $\mathcal{O}$ qui est un supplémentaire de $I$. Ce supplémentaire ne dépend que de l'idéal initial in $I=$ in $m^{k}$, et donc $\Delta(I)=\Delta\left(m^{k}\right)$. On en déduit que $h \in m^{k} \cap \Delta\left(m^{k}\right)=\{0\}$, d'où $h=0$, et $g \in I$.

Remarque. Soit $A$ un anneau local noethérien régulier contenant un corps, et soit $x_{1}, \ldots, x_{d}$ un système régulier de paramètres de $A$. Si $\hat{A}$ désigne le 
complété de $A$, on a alors $\hat{A} \simeq K\left[\left[x_{1}, \ldots, x_{d}\right]\right.$ avec $K \simeq A / m$ par le théorème de structure de Cohen, cf. [M2], Th. 29.7. Si l'on suppose alors de plus que $A$ est stable par les dérivations $\partial_{x_{f}}$, le résultat ci-dessus (i.e., le lemme 2) est vrai dans $A$ : on montre comme plus haut le lemme 2 dans $\hat{A}$, et le résultat pour $A$ découle du fait que $\mathbb{D}_{m \hat{A}}$ est engendré par les $x_{l} \partial_{\lambda_{\jmath}}$, et que les $x_{i} \partial_{r_{\jmath}}$ appartiennent à $\mathbb{D}_{m}$ par hypothèse: l'inclusion $\mathbb{D}_{m} \subset \mathbb{D}_{I}$ implique alors $\mathbb{D}_{m \hat{A}} \subset \mathbb{D}_{I \hat{A}}$, et on utilise la platitude de $\hat{A}$ sur $A$.

Soit maintenant $\wp$ un idéal premier de $\mathcal{O}$, et $A=\mathcal{O}_{\wp}$ le localisé de $\mathcal{O}$ en $\wp$. L'anneau $A$ est local régulier, et il vérifie la condition ci-dessus, comme il résulte par exemple de [M2], Th. 30.6, p.237. On peut donc lui appliquer la remarque, ce qui donne:

Corollaire. Soient $\wp$ un idéal premier de $\mathcal{C}$ et I un idéal de $\mathcal{O}_{\wp}$. Notons $\mathbb{D}^{\prime}$ l'algèbre des dérivations de $\mathcal{O}_{\S}$. Alors $\mathbb{D}_{\wp^{\prime} \mathcal{C}^{\prime}}^{\prime} \subset \mathbb{D}_{I}^{\prime}$ si et seulement si $I=\wp^{k} \mathcal{O}_{\wp}$ pour un entier $k>0$.

Si $\wp$ est un idéal premier de $\mathcal{O}$, nous noterons $\wp^{(k)}$ la $k$-ième puissance symbolique de $\wp$, i.e., l'idéal $\wp^{(k)}=\wp^{k} \mathcal{O}_{\wp} \cap \mathcal{O}$. Pour $f \in \mathcal{O}$, on a $f \in \wp^{(k)}$ si et seulement si il existe $a \in \mathcal{O} \backslash \wp$ tel que $a f \in \wp^{k}$.

Proposition 1. Soit $\wp \subset \mathcal{O}$ un idéal premier, $I \subset \wp$ un idéal $\wp$-primaire. Les conditions suivantes sont équivalentes:

(a) $\mathbb{D}_{I}=\mathbb{D}_{\wp}$.

(b) Il existe un entier $k>0$ tel que $I=\wp^{(k)}$.

Démonstration. $\quad(\mathrm{a}) \Rightarrow(\mathrm{b})$. Prouvons d'abord que pour tout $D \in \mathbb{D}^{\prime}$ il existe $a \in \mathcal{O} \backslash \wp$ tel que $a D \in \mathbb{D}$. Supposons que $\mathcal{C}=\mathbb{R}\left\{x_{1}, \ldots, x_{n}\right\}$, et considérons $a \in \mathbb{O} \backslash \wp$ tel que $a D x_{i} \in \mathcal{O}$, pour $1 \leq i \leq n$. Alors pour tout monôme $x^{\alpha}$, il suit que $a D x^{\alpha} \in \mathcal{O}$. On en déduit que si $f \in \mathcal{O}, a D f \in \hat{\mathcal{O}} \cap \mathcal{O}_{\wp}=\mathcal{C}$, donc $a D \in \mathbb{D}$.

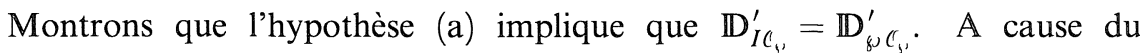
lemme $1(\mathrm{~b})$ il suffit de montrer que $\mathbb{D}_{\wp_{0},}^{\prime} \subset \mathbb{D}_{I c_{0},}^{\prime}$. Soit donc $D \in \mathbb{D}_{\wp \jmath}, a \in \mathcal{Q} \backslash \wp$ tel que $a D \in \mathbb{D}$. On a alors $a D \in \mathbb{D}_{\wp}=\mathbb{D}_{I}$. Si maintenant $f \in I O_{\wp}$, il existe $b \in \mathcal{O} \backslash \wp$ tel que $b f \in I$. Ceci donne $a D(b f) \in I$. Comme $a D(b f)=a f D b+$ $a b D f$, on en déduit $a b D f \in I \mathcal{O}_{\wp}$, et donc $D f \in I \mathcal{O}_{\wp}$, puisque $a b \notin \wp$. D'après le corollaire, $I \mathcal{O}_{\wp}=\wp^{k} \mathcal{O}_{\wp}$ pour un entier $k>0$. On a donc $I \subset \wp^{k} \mathcal{O}_{\wp} \cap \mathcal{O}=\wp^{(k)}$, ce qui implique $I=\wp^{(k)}$, puisque $I$ est supposé $\wp$-primaire (cf. [A-M], Thm. 4.8: pour tout idéal $I \wp$-primaire, on a l'égalité $\left.I \mathcal{O}_{\wp} \cap \mathcal{O}=I\right)$.

(b) $\Rightarrow$ (a). On a $\mathbb{D}_{I} \subset \mathbb{D}_{\wp}$ puisque par hypothèse $\wp=\sqrt{I}$. Réciproquement, soient $D \in \mathbb{D}_{\wp}, f \in \wp^{(k)}$. Par définition de la puissance symbolique, il existe $a \in \mathcal{O} \backslash \wp$ tel que $a f \in \wp^{k}$. Comme $\mathbb{D}_{\wp}=\mathbb{D}_{\wp^{k}}$, on a $D \in \mathbb{D}_{\wp^{k}}$, et donc $D(a f) \in \wp^{k}$. On a donc $a^{2} D f=a D(a f)-a f D a \in \wp^{k}$, d'où $D f \in \wp^{(k)}=I$. 
Théorème 1. Soit $I \subset \mathcal{O}$ un idéal sans composantes immergées, et notons $\wp_{i}$ ses idéaux premiers associés. Alors les conditions suivantes sont équivalentes:

(a) $\mathbb{D}_{I}=\mathbb{D}_{\sqrt{I}}$.

(b) $I=\wp_{1}^{\left(k_{1}\right)} \cap \cdots \cap \wp_{s}^{\left(k_{s}\right)}$ pour des entiers $k_{i}>0$.

Démonstration. $\quad(\mathrm{a}) \Rightarrow(\mathrm{b})$. Posons $I_{i}=I \mathcal{O}_{\wp_{1}} \cap \mathcal{O}$ de sorte que $I=I_{1} \cap \cdots$ $\cap I_{s}$ avec les $I_{i} \wp_{i}$-primaires. Supposons $\mathbb{D}_{I}=\mathbb{D}_{\sqrt{I}}$. La proposition 1 implique qu'il suffit de prouver que $\mathbb{D}_{I_{l}}=\mathbb{D}_{\wp_{l}}$ pour tout $i$. L'inclusion $\mathbb{D}_{I_{l}} \subset \mathbb{D}_{\wp_{l}}$, vient du lemme 1(a), puisque $\wp_{l}=\sqrt{I}_{i}$. Montrons l'inclusion inverse par exemple dans le cas $i=s$, i.e., que $\mathbb{D}_{\wp_{s}} \subset \mathbb{D}_{I_{\varsigma}}$. Soit $D \in \mathbb{D}_{\wp_{s}}$, et choisissons $a \in \wp_{1} \cap \cdots \cap \wp_{s-1} \backslash \wp_{s}$. Alors $a D \in \mathbb{D}_{\wp_{1}} \cap \cdots \cap \mathbb{D}_{\wp_{s}}$, d'où $a D \in \mathbb{D}_{\sqrt{I}}$ par le lemme 1(b). L'hypothèse implique $a D \in \mathbb{D}_{I}$ et le lemme $1(\mathrm{c})$ donne $a D \in \mathbb{D}_{I_{s}}$. Comme $a \notin \wp_{s}$, on en déduit $D \in \mathbb{D}_{I_{s}}$, puisque $I_{s}$ est $\wp_{s}$-primaire (on a $I_{s}=I_{s} \mathcal{O}_{\wp_{s}} \cap \mathcal{O}$ comme rappelé plus haut).

(b) $\Rightarrow$ (a). On a $\mathbb{D}_{I} \subset \mathbb{D}_{\sqrt{I}}$ par le lemme 1(a). Réciproquement, on a $\mathbb{D}_{\sqrt{I}}=\cap \mathbb{D}_{\wp_{1}}($ lemme $1(\mathrm{c}))=\cap \mathbb{D}_{\wp_{1}^{\left(k_{t}\right)}}($ proposition 1$) \subset \mathbb{D}_{\bigcap_{\wp_{1}}^{\left(k_{l}\right)}}($ lemme $1(\mathrm{~b}))=$ $\mathbb{D}_{I}$ (par hypothèse).

Remarque. L'assertion du théorème 1 n'est plus nécessairement vraie si $I$ possède des composantes immergées. Considérons par exemple dans $\mathbf{R}\{x, y\}$ l'idéal $I=(y) \cap\left(x^{2}, x y, y^{2}\right)=(y) \cap(x, y)^{2}=\left(x y, y^{2}\right)$. On a $\sqrt{I}=(y)$, et $\partial_{x} \in$ $\mathbb{D}_{\sqrt{I}}$, alors que $\partial_{y} \notin \mathbb{D}_{I}$.

\section{Rappels sur les Germes Analytiques Réels}

Un germe d'espace analytique en $0 \in \mathbf{R}^{n}$ sera un couple $\left(X, \mathcal{O}_{X}\right)$, où $\mathcal{O}_{X}=\mathcal{O} / I$ pour un idéal $I$ de $\mathcal{O}$, et où $X$ est l'ensemble des zéros de $I$. Nous identifierons toujours un germe d'espace analytique avec un représentant suffisamment petit. Ainsi on pourra parler d'un point $x$ de $X$ etc. Si $\left(X, \mathcal{O}_{X}\right)$ est un germe réel, son complexifié est le germe $\left(X^{\mathrm{C}}, \mathcal{O}_{X^{\mathrm{c}}}\right)$ défini par l'idéal $I^{\mathbf{C}}=I \otimes \mathbf{C}=I 0^{\mathbf{C}}$. On dit que $X$ est réduit si $I$ est réduit, i.e., égal à sa racine $\sqrt{I}$. Si $X$ est réduit, il en est de même de $X^{\mathbf{C}}[\mathrm{Ru}, 5.3]$. Si $I$ est un idéal de $\mathcal{O}$, nous noterons $\sqrt[\mathrm{R}]{I}$ sa racine réelle. Par définition,

$$
\sqrt[\mathrm{R}]{I}=\left\{f \in \mathcal{O} \mid f^{2 m}+\sum a_{1}^{2} \in I \text { pour un } m \in \mathbf{N} \text { et } \operatorname{des} a_{l} \in \mathcal{O}\right\} .
$$

Notant $I(X)$ l'idéal des fonctions qui s'annulent sur $X$ on a $I(X)=\sqrt[\mathrm{R}]{I}[\mathrm{R}, \mathrm{Ru}]$. On dit que $I$ est réel, si $I=\sqrt[\mathrm{R}]{I}$.

Soit $X$ un germe réduit. Le lieu singulier complexe $\left(\operatorname{Sing} X^{\mathbf{C}}, \mathcal{O}^{\mathbf{C}} / J\right)$ sera toujours considéré avec sa structure réduite. L'idéal $J$ est alors invariant par la conjuguaison complexe [H, Remark 5.6], i.e., engendré par des germes réels (l'idéal $J$ est en effet la racine d'un idéal défini uniquement par des générateurs réels). On définit alors $\operatorname{Sing} X$ comme la partie réelle de $\operatorname{Sing} X^{\mathrm{C}}$ (avec comme 
idéal la partie réelle de $J$ ). C'est donc un germe analytique réel réduit. Remarquons que l'on peut avoir (ensemblistement) $X=\operatorname{Sing} X$ alors que $\operatorname{Sing} X^{\mathrm{C}}$ est toujours strictement inclus dans $X^{\mathrm{C}}$. C'est par exemple le cas pour le germe de $\left(\mathbf{R}^{2}, 0\right)$ défini par l'idéal $\left(x^{2}+y^{2}\right)$.

Lemme 3. Soit $X$ un germe défini par un idéal réduit I. Considérons les propriétés suivantes:

(a) $I=\sqrt[\mathrm{R}]{I}$.

(b) L'inclusion $\operatorname{Sing} X \subset X$ est stricte.

Alors (a) implique (b), et (b) implique (a) dans le cas où I est un idéal premier.

Démonstration. Dans le cas où $I$ est un idéal premier, l'assertion se trouve dans $[\mathrm{Ru}, 4.3, \mathrm{p} .80]$. Montrons que (a) implique (b) dans le cas où $I$ est réduit; $I$ étant un idéal réel, tous les idéaux premiers minimaux contenant $I$ sont réels $[\mathrm{R}, 1.1]$. La condition (b) suit immédiatement en appliquant le lemme à l'idéal d'une composante irréductible de $X$ de dimension maximale.

On peut alors définir par récurrence l'espace analytique réduit $\operatorname{Sing}^{k} X$ par $\operatorname{Sing}^{k} X=\operatorname{Sing}\left(\operatorname{Sing}^{k-1} X\right)$ pour $k \geq 1$, posant $\operatorname{Sing}^{0} X=X$. Si $x \in X$, il existe un unique $k$ tel que $x \in \operatorname{Sing}^{k} X \backslash \operatorname{Sing}^{k+1} X$.

Pour un germe $X \subset\left(\mathbb{R}^{n}, 0\right)$, on dispose de deux topologies: La topologie 'Euclidienne', induite par la topologie de $\mathbb{R}^{n}$, notée avec l'indice $e$, et la topologie 'de Zariski', notée avec l'indice $z$, et dont les fermés sont les zéros des idéaux de $\mathscr{C} / I$. Soit $x \in X$. Nous noterons $\mathcal{C}_{X}$, l'anneau local de $X$ en $x: \mathcal{C}_{X, 1}=\mathcal{C}_{\mathbf{R}^{n}, 、} / I_{1}$, et $m_{X, \lambda}$ l'idéal maximal de $\mathbb{C}_{X, 1}$. On note $\mathrm{T}: X=$ $\left(m_{X, 、} / m_{X, 1}^{2}\right)^{*}$ l'espace tangent de Zariski à $X$ en $x$.

Si $x \notin \operatorname{Sing} X$, on dit que $x$ est régulier; ceci équivaut à dire que l'anneau $\mathfrak{C}_{X .}$, est un anneau local régulier. L'ensemble des points réguliers est noté $\operatorname{Reg} X$. L'ensemble des points lisses de $X$ est l'ensemble des points au $e$ voisinage desquels $X$ est une variété analytique lisse. Cet ensemble contient $\operatorname{Reg} X$. En un point $x \in X$ lisse, l'espace tangent $\mathrm{T}_{\imath}^{e} X \subset \mathbb{R}^{n}$ a la même dimension que $\mathrm{T}: X$ si et seulement si $x$ est régulier. Par exemple, si $X$ est le "parapluie de Whitney", défini dans $\left(\mathbb{R}^{3}, 0\right)$ par l'équation $x^{2}-y^{2} z=0$, les points $(0,0, z)$ avec $z<0$ sont des points lisses qui ne sont pas réguliers, alors que les points $(x, y, z)$ de $X$ avec $z>0$ et $x^{2}+y^{2} \neq 0$ sont réguliers, donc lisses de dimension 2 .

Lemma 4. Soient $X$ un germe réduit défini par un idéal I et $Y$ le germe défini par $\sqrt[\mathrm{R}]{I}$.

(a) L'ensemble Reg $Y$ est z-dense dans $Y$.

(b) Soit $Z$ une composante irréductible de $Y$ et soit $k$ le plus grand entier tel que $Z \subset \operatorname{Sing}^{k} X . \quad$ Il existe un $z$-ouvert $U$ non vide de $Z$, tel que pour $x \in U$ les germes en $x$ de $Z$ et $\operatorname{Sing}^{k} X$ coüncident. 
Démonstration. La propriété (a) est vraie pour chaque composante irréductible de $Y$ par le lemme 3(c) car si $J$ est un idéal réel, tous les idéaux premiers minimaux contenant $J$ sont réels $[\mathrm{R}, 1.1]$. Pour (b), comme $Z$ n'est pas contenu dans $\operatorname{Sing}^{k+1} X$, le $z$-ouvert $U=\operatorname{Reg} Z \backslash \operatorname{Sing}^{k+1} X$ de $Z$ est non vide (d'après (a)), et tout point $x$ de $U$ est régulier pour $Z$ et $\operatorname{Sing}^{k} X$. Au $e$ voisinage de $x, Z$ et $\operatorname{Sing}^{k} X$ sont deux germes lisses de même $e$-dimension (car ensemblistement $Z$ et $\operatorname{Sing}^{k} X$ coïncident au voisinage de $x$, puisque on a les inclusions $Z \subset \operatorname{Sing}^{k} X \subset Y$, et que ensemblistement $Z=Y$ au voisinage de $x$ ), donc ils coïncident.

\section{Idéaux Réels Invariants}

Nous allons dans ce paragraphe donner quelques propriétés élémentaires de $\mathbb{D}_{I}$, démontrées dans $[\mathrm{H}-\mathrm{M}]$ pour les germes analytiques complexes, et dont nous aurons besoin dans le cas réel pour étudier les rapports entre $\mathbb{D}_{I}$ et $\mathbb{D}_{\sqrt[R]{I}}$.

Les éléments de $\mathbb{D}$ s'identifient aux germes de champs de vecteurs sur $\left(\mathbb{R}^{n}, 0\right)$, et nous noterons $D(x)$ la valeur du champ $D \in \mathbb{D}$ au point $x$.

Lemme 5. Soient I un idéal réel de $\mathcal{C}$ et $X$ le germe défini par $I$. Pour $D \in$ $\mathbb{D}_{I}$ on a $D(x) \in \mathrm{T}_{1}^{z} X$ pour tout point $x \in X$. Réciproquement, si $D(x) \in \mathrm{T}_{\imath}^{z} X$ pour tout point $x$ d'un ensemble $z$-dense de $X, D \in \mathbb{D}_{I}$.

Le démonstration de $[\mathrm{H}-\mathrm{M}]$, Proposition 2.1, se transpose sans changement au cas réel. (On considère l'application $f:\left(\mathbf{R}^{n}, 0\right) \rightarrow\left(\mathbf{R}^{n}, 0\right)$ définie par des generateurs $f_{l}$ de $I$. L'espace $\mathrm{T}_{\imath}^{z} X$ s'identifie alors à $\left(\mathrm{T}_{、} f\right)^{-1}(0) \subset \mathbb{R}^{n}$. Si $D=\sum a_{l} \partial_{\mathrm{i}_{1}}, x \in X$, on a $D(x) \in \mathrm{T}_{r}^{z} X$ si et seulement si $\sum a_{l} \partial_{\mathbf{}_{l}} f(x)=\left(\mathrm{T}_{、} f\right)\left(a_{1}(x), \ldots, a_{n}(x)\right)=0$. Ceci est vrai pour $x$ dans un ensemble $z$-dense de $X$ si et seulement si $D f_{J} \in I_{X}$, c'est à dire si et seulement si $\left.D\left(I_{X}\right) \subset I_{X}\right)$.

Remarquons que si $X$ est irréductible, tout $e$-ouvert non vide de $\operatorname{Reg} X$ est $z$-dense.

Proposition 2. Soient $I \subset J$ deux idéaux réduits dans $\mathcal{O}$. Alors $\mathbb{D}_{I}=\mathbb{D}_{J}$ si et seulement si $I=J$.

Démonstration. On a $I=J$ si et seulement $I 0^{\mathbf{C}}=J \Theta^{\mathbf{C}}$. Le fait que $I \Theta^{\mathbf{C}}$ et $J C^{(} \mathbf{C}$ soient réduits et le lemme suivant montrent qu'il suffit de montrer la proposition 2 dans le cas complexe.

Lemme 6. Soit $I \subset \mathcal{O}$ un idéal. Alors $\mathbb{D}_{I} \otimes \mathbb{C}=\mathbb{D}_{I \otimes C}^{C}$.

Démonstration. L'inclusion $\mathbb{D}_{I} \otimes \mathbf{C} \subset \mathbb{D}_{I \otimes \mathbf{C}}^{\mathbf{C}}$ est évidente, puisque $I \otimes \mathbf{C}$ est engendré par $I$. Montrons l'inclusion inverse. On définit le conjugué $\bar{D}$ d'une dérivation $D$ de $\mathbb{D}^{\mathbf{C}}$ par la formule $\bar{D}(g)=\overline{D(g)}$ pour tout $g \in \mathcal{C}^{\mathrm{C}}$, ce qui 
donne une involution sur le $\mathcal{O}^{\mathrm{C}}$-module des dérivations. Soit $D \in \mathbb{D}_{I \otimes \mathbf{C}}^{\mathrm{C}}$, et $I=$ $\left(g_{1}, \ldots, g_{s}\right)$. Posons $D g_{i}=\sum a_{i j} g_{j}$, avec $a_{i j} \in \mathcal{O}^{\mathbf{C}}$ par hypothèse. On a $\bar{D} g_{i}=$ $\sum \overline{a_{i j}} g_{J}$, d'où $D+\bar{D} \in \mathbb{D}_{I}$ et $\frac{1}{i}(D-\bar{D}) \in \mathbb{D}_{I}$. Ceci implique $D \in \mathbb{D}_{I} \otimes \mathbb{C}$, puisque $D=\frac{1}{2}(D+\bar{D})+\frac{1}{2 i}(D-\bar{D})$.

Pour montrer la proposition 2 , on suppose donc que l'on est dans $\mathcal{O}^{\mathbf{C}}$, et on suit $[\mathrm{H}-\mathrm{M}]$, proposition 5.2. Soient $X$ et $Y$ les germes définis par $I$ et $J$. Supposons $I \neq J$, et soit $f \in J \backslash I$. Si $x_{1}, \ldots, x_{n}$ forment un système régulier de paramètres de $\mathcal{O}$, les champs de vecteurs $f \partial_{x_{1}}, \ldots, f \partial_{x_{n}}$ appartiennent à $\mathbb{D}_{J}$. Comme les germes sont complexes, il existe un point lisse $x$ de $X \backslash Y$ tel que $f(x) \neq 0$. Choisissons $i$ tel que $\partial_{x_{t}}(x) \notin \mathrm{T}_{x}^{z}(Y)$. Par le lemme 5, on a que $f \partial_{x_{l}} \notin \mathbb{D}_{J}$ ce qui est absurde puisque $f \in J$.

La proposition 2 implique en particulier que si $I$ est un idéal de $\mathcal{O}, \mathbb{D}_{\sqrt{I}}=$ $\mathbb{D}_{\sqrt[\mathrm{R}]{I}}$ si et seulement si $\sqrt{I}=\sqrt[\mathrm{R}]{I}$. Plus généralement, on a le résultat suivant:

Théorème 2. Pour tout idéal I de $\mathcal{O}$ on a $\mathbb{D}_{I} \subset \mathbb{D}_{\sqrt{I}} \subset \mathbb{D}_{\sqrt[R]{I}}$.

Démonstration. La première inclusion est le lemme 1(a). Pour montrer la deuxième, supposons $I$ réduit et considérons $D \in \mathbb{D}_{I}$. Soient $X$ et $Y$ les germes définis par $I$ et $\sqrt[\mathrm{R}]{I}$. Pour montrer que $D \in \mathbb{D}_{\sqrt[R]{I}}$, il suffit d'établir, par le lemme 5, que $D(x) \in \mathrm{T}_{x}^{z} Y$ pour $x$ dans un ensemble $z$-dense de $Y$. Il suffit pour cela que pour chaque composante irréductible $Z$ de $Y, D(x) \in \mathrm{T}_{x}^{z} Z$ pour $x$ dans un z-ouvert non vide de $Z$. Soit donc $Z$ une composante de $Y$, et soit $k$ le plus grand entier tel que $Z \subset \operatorname{Sing}^{k} X$.

Lemme 7. Soit $(X, \mathcal{O} / I)$ un germe analytique réduit, et $J \subset \mathcal{O}$ l'idéal de Sing $X$. On a $\mathbb{D}_{I} \subset \mathbb{D}_{J}$.

Démonstration. Soit $D \in \mathbb{D}_{I}$, et soit $X^{\mathrm{C}}$ le complexifié de $X$. Alors $J^{\mathrm{C}}=$ $J \mathcal{O}^{\mathbf{C}}$ est l'idéal de $\operatorname{Sing} X^{\mathbf{C}}$. On sait alors par $[\mathrm{H}-\mathrm{M}]$, proposition 5.1(a), que $D\left(J^{\mathrm{C}}\right) \subset J^{\mathrm{C}}$, ce qui implique $D(J) \subset J$, puisque $J=J \mathcal{O}^{\mathbf{C}} \cap \mathcal{O}$ par platitude de $\mathcal{O}^{\mathrm{C}} \operatorname{sur} \mathcal{O}$.

Montrons maintenant le théorème 2: les lemmes 5 et 7 impliquent que $D(x)$ appartient à $\mathrm{T}_{x}^{z}\left(\operatorname{Sing}^{k} X\right)$ pour $x$ dans un $z$-ouvert de $Z$ (en fait, c'est vrai pour tout $\left.x \in \operatorname{Sing}^{k} X\right)$. Comme $\mathrm{T}_{x}^{z} Z=\mathrm{T}_{x}^{z}\left(\operatorname{Sing}^{k} X\right)$ dans un $z$-ouvert non vide de $Z$ par le lemme 4 , la deuxième inclusion est démontrée.

\section{Sous-algèbres Maximales de $\mathbb{D}_{Y}$}

Les résultats de ce paragraphe (proposition 3 ci-dessous) seront utilisés dans la démonstration du théorème 3 au paragraphe suivant. 
On note $A^{\mathrm{C}}$ l'algèbre complexifiée d'une algèbre réelle $A$. Rappelons que pour une inclusion $A \subset B$ d'algèbres de Lie la série des transporteurs de $A$ dans $B$ est définie comme suit.

$$
\begin{gathered}
A^{[1]}=\{D \in A,[D, B] \subset A\}, \\
A^{[l]}=\left\{D \in A^{[l-1]},[D, B] \subset A^{[l-1]}\right\}, \\
A^{[\infty]}=\bigcap A^{[l]} .
\end{gathered}
$$

On dit que $A$ est balancée dans $B$ si $A^{[\infty]}=0$ et $A^{[2]} \neq 0$. Comme le crochet commute avec la complexification, et que $A \subset B$ si et seulement si $A^{\mathbf{C}} \subset B^{\mathbf{C}}, A$ est balancée dans $B$ si et seulement si $A^{\mathbf{C}}$ l'est dans $B^{\mathbf{C}}$.

On se place maintenant dans le cadre des ensembles algébriques, notant par $\mathbb{R}[X]$ (resp. $\mathbb{C}[X]$ ) l'anneau des polynômes à $n$ variables. Si $X$ est un sousensemble algébrique de $\mathbf{R}^{n}$ (resp. $\mathbf{C}^{n}$ ), nous noterons $I_{X}$ l'idéal des polynômes nuls sur $X$, et $\mathbb{D}_{X}=\mathbb{D}_{I_{\lambda}}$. Soit $Y \subset \mathbb{R}^{n}\left(\right.$ resp. $\mathbb{C}^{n}$ ) fixée. Pour $A \subset \mathbb{D}_{Y}$ une sous-algèbre de Lie, nous poserons $I_{A}=\left\{g \in \mathcal{O}, g \mathbb{D}_{Y} \subset A\right\}$ et noterons $X_{A} \subset \mathbf{R}^{n}$ (resp. $\mathbb{C}^{n}$ ) le sous-ensemble algébrique défini par l'idéal $I_{A}$. Dans le cas réel, on a alors $\left(X_{A}\right)^{\mathrm{C}}=X_{A} \mathrm{c}$. Si $X$ et $X^{\prime}$ sont deux ensembles algébriques, on pose $\mathbb{D}_{X, X^{\prime}}=\mathbb{D}_{X} \cap \mathbb{D}_{X^{\prime}}$. Nous emploierons dans ce paragraphe plutôt le mot champ que le mot dérivation pour désigner un élément de $\mathbb{D}$.

Commençons par caractériser les sous-algèbres maximales balancées (i.e., maximales parmi les sous-algèbres balancées) de codimension finie de $\mathbb{D}_{Y}$ dans le cas complexe.

Lemme 8. Soit $Y \subset \mathbb{C}^{n}$ un sous-ensemble algébrique, $Y \neq \mathbb{C}^{n}$, et $A \subset \mathbb{D}_{Y}$ une sous-algèbre de Lie. Alors les conditions suivantes sont équivalentes:

(a) A est maximale balancée de codimension finie dans $\mathbb{D}_{Y}$.

(b) $A=\mathbb{D}_{Y, P}$ pour un point $P \in \mathbb{C}^{n} \backslash Y$.

Démonstration. Ce résultat, déjà prouvé par Omori $[\mathrm{O}]$, est un cas particulier de la théorie plus générale de $[\mathrm{H}-\mathrm{M}]$ sur la caractérisation des sousalgèbres maximales balancées. Pour la commodité du lecteur, nous donnons ici les indications nécessaires pour obtenir une démonstration directe dans le cas de codimension finie.

(a) $\Rightarrow$ (b). Soit $X_{A} \subset \mathbb{C}^{n}$ le sous-ensemble algébrique défini par l'idéal $I_{A}$, et considérons l'algèbre $\mathbb{D}_{Y, X_{4}}$ (c'est l'algèbre tangente à $Y \cup X_{A}$ ). On a immédiatement, par définition de $I_{A}$ et le lemme 1(a), les inclusions

$$
A \subset \mathbb{D}_{I_{A}} \subset \mathbb{D}_{\sqrt{I_{A}}} \subset \mathbb{D}_{Y}
$$

Les propositions $6.2(\mathrm{c})$ et $5.2 \mathrm{de}[\mathrm{H}-\mathrm{M}]$ appliquées à l'algèbre balancée $A$ montrent que la troisième inclusion est stricte. L'algèbre $\mathbb{D}_{Y, X_{4}}$ est de codimension finie dans $\mathbb{D}_{Y}$, donc, par $[\mathrm{H}-\mathrm{M}]$ proposition $4.2, X_{A}$ est une réunion finie de points. 
Si ce nombre était plus grand que $1, \mathbb{D}_{Y, X_{A}}$ ne serait pas maximale, car incluse dans $\mathbb{D}_{Y, \tilde{X}_{4}}$, où $\tilde{X}_{A}=X_{A}$ moins un point. On a donc $X_{A}=\{P\}$, et $P \notin Y$ par $[\mathrm{H}-\mathrm{M}]$, proposition $6.2(\mathrm{c})$.

(b) $\Rightarrow(a)$. Par $[\mathrm{H}-\mathrm{M}]$, proposition. $6.1(\mathrm{c})$, on voit que $\mathbb{D}_{Y, P}$ est balancée dans $\mathbb{D}_{Y}$, et il est clair que sa codimension est finie. Il reste à montrer qu'elle est maximale (balancée). Soit donc $A$ une algèbre balancée telle que $\mathbb{D}_{Y, P} \subset A \subset$ $\mathbb{D}_{Y}$. On a alors comme plus haut

$$
\mathbb{D}_{Y, P} \subset A \subset \mathbb{D}_{Y . X_{A}} \subset \mathbb{D}_{Y}
$$

avec $X_{A}$ une réunion finie de points, dont un au moins non dans $Y$. On a alors $X_{A}=\{P\}$ par $[\mathrm{H}-\mathrm{M}]$, proposition 5.2 , ce qui donne $A=\mathbb{D}_{Y, P}$, et $A$ est donc maximale balancée.

Dans la cas réel, on a la proposition suivante:

Proposition 3. Soient $Y \subset \mathbb{R}^{n}$ un sous-ensemble algébrique, $Y \neq \mathbb{R}^{n}, A$ une sous-algèbre de Lie de codimension finie $s$ de $\mathbb{D}_{Y}$, qui est balancée et maximale parmi les sous-algèbres balancées de $\mathbb{D}_{Y}$. Alors les conditions suivantes sont équivalentes.

(a) $A^{\mathbf{C}}$ est maximale balancée dans $\mathbb{D}_{Y}^{\mathbf{C}}$.

(b) $X_{A}=\{P\}$, où $P$ est un point $P \in \mathbb{R}^{n} \backslash Y$.

(c) $X_{A} \neq \varnothing$.

(d) $s=n$.

De plus, $A^{\mathbf{C}}$ est non maximale (balancée) dans $\mathbb{D}_{Y}^{\mathbf{C}}$ si et seulement si $X_{A}^{\mathbf{C}}=$ $\{P, \bar{P}\}$, avec $P \notin \mathbb{R}^{n}, P \notin Y^{\mathbf{C}}(\bar{P}$ est le conjugué de $P)$, ou encore, si et seulement si $s=2 n$.

Démonstration. Remarquons que $\mathbb{D}_{Y}^{\mathrm{C}}=\mathbb{D}_{Y^{\mathrm{c}}}^{\mathrm{C}}$, car on a $I_{Y^{\mathrm{c}}}=\left(I_{Y}\right)^{\mathrm{C}}$, par le lemme 3 .

(a) $\Rightarrow$ (b). On sait que $X_{A^{\mathrm{c}}}=\{P\}$, avec $P \in \mathbb{C}^{n} \backslash Y^{\mathbf{C}}$, par le lemme 8. De plus, $P$ est invariant par la conjuguaison complexe puisque $A^{\mathrm{C}}$ l'est. $^{\prime}$

(b) $\Rightarrow$ (c), (d). Evident.

(d) $\Rightarrow$ (a). L'hypothèse implique que $A^{\mathbf{C}}$ est de codimension $n$ dans $\mathbb{D}_{Y}^{\mathbf{C}}$. Comme toute sous-algèbre balancée de $\mathbb{D}_{Y}^{\mathrm{C}}$ est de codimension au moins $n$ par les propositions 4.2 et 7.1 de $[\mathrm{H}-\mathrm{M}]$, on en déduit que $A^{\mathbf{C}}$ est maximale balancée dans $\mathbb{D}_{Y}^{\mathbf{C}}$.

(c) $\Rightarrow$ (a). Supposons que $A^{\mathrm{C}}$ ne soit pas maximale dans $\mathbb{D}_{Y}^{\mathrm{C}}$. Le lemme 8 implique que $X_{A}^{\mathrm{C}}=\left\{P_{1}, \ldots, P_{k}\right\}$ avec $k \geq 2$ et que au moins un des $P_{l}$ n'est pas contenu dans $Y^{\mathrm{C}}$, disons $P_{1}$. Supposons d'abord $k=2$. Deux cas peuvent alors se présenter: soit $P_{1}=\bar{P}_{2}$ avec $P_{1} \notin \mathbb{R}^{n}$ et donc $X_{A}=\varnothing$, soit $P_{1} \in \mathbb{R}^{n}$ et $P_{2} \in \mathbb{R}^{n}$. Montrons que dans ce cas $A \subset \mathbb{D}_{Y}$ n'est pas maximale. La sous-algèbre $\mathbb{D}_{Y, P_{1}}^{\mathrm{C}}$ de $\mathbb{D}_{Y}^{\mathrm{C}}$ formée des champs tangents à $Y$ et nuls en $P_{1}$ vérifie $A^{\mathrm{C}} \subset \mathbb{D}_{Y, P_{1}}^{\mathrm{C}} \subset \mathbb{D}_{Y}^{\mathrm{C}}$, les inclusions étant strictes, cf. le lemme 8 . Il existe 
donc un champ $D \in \mathbb{D}_{Y}^{\mathrm{C}}$ tel que $D\left(P_{1}\right)=0$ et $D\left(P_{2}\right) \neq 0$. Alors $D+\bar{D}$ et $\frac{1}{i}(D-\bar{D})$ vérifient les mêmes propriétés et sont réels, ce qui donne les inclusions strictes $A \subset \mathbb{D}_{Y, P_{1}} \subset \mathbb{D}_{Y}$.

Dans le cas $k>2$ on peut écrire $X_{A}^{\mathbf{C}}=Q_{1} \cup Q_{2}$ où $Q_{i}$ désigne une réunion finie de points de $Y^{\mathrm{C}}$ stable par conjuguaison. On applique exactement le même raisonnement que pour $k=2$ et trouve que $Q_{1}=\left\{P_{1}, \bar{P}_{1}\right\}$ et $Q_{2}=\varnothing$. Ceci prouve $(\mathrm{c}) \Rightarrow(\mathrm{a})$ ainsi que la fin de l'énoncé.

On a de manière analogue, dans le cas non nécessairement de codimension finie:

Proposition 4. Soit $A \subset \mathbb{D}_{Y}$ une sous-algèbre de Lie balancée maximale. Les propriétés suivantes sont équivalentes:

(a) $A^{\mathbf{C}} \subset \mathbb{D}_{Y}^{\mathbf{C}}$ est maximale.

(b) $X_{A}$ est une sous-variété irréductible de $\mathbf{R}^{n}$ avec $\operatorname{Sing} X_{A} \subset Y$ mais $X_{A} \not \subset Y$. De plus non (a) est équivalent à $X_{A}^{\mathrm{C}}=Z \cup \bar{Z}$, avec $Z \neq \bar{Z}, Z \cap \bar{Z} \subset Y, Z \not \subset Y$.

Démonstration. Montrons d'abord que si $A \subset \mathbb{D}_{Y}$ est balancée maximale, alors $\sqrt{I}_{A}$ est réelle (i.e., $\sqrt{I}_{A}=\sqrt[\mathrm{R}]{I_{A}}$ ). En effet, si $\sqrt{I}_{A}$ n'est pas réelle, l'inclusion $\sqrt{I}_{A} \subset \sqrt[\mathrm{R}]{I_{A}}$ est stricte. On a alors une chaîne d'inclusions d'algèbres (théorème 2):

$$
A \subset \mathbb{D}_{I_{A}} \subset \mathbb{D}_{\sqrt[R]{I_{A}}} \subset \mathbb{D}_{Y}
$$

et l'inclusion $\mathbb{D}_{\sqrt{I}_{A}} \subset \mathbb{D}_{\mathbb{R}_{I_{A}}}$ est stricte d'après la proposition 2. Cela contredit donc la maximalité de $A$. La démonstration de la proposition 4 est ensuite pratiquement la même que celle de la proposition 3 .

\section{Caractérisation des Variétés par Leurs Algèbres Tangentes}

Voici les analogues réels des résultats principaux de [H-M].

Considérons $X, Y \subset \mathbf{R}^{n}$ deux sous-ensembles algébriques isomorphes par un isomorphisme restriction d'un isomorphisme algébrique $\phi: \mathbf{R}^{n} \rightarrow \mathbf{R}^{n}$. L'automorphisme correspondant

$$
\phi^{*}: \mathbf{R}[X] \rightarrow \mathbf{R}[X], \quad f \mapsto f \circ \phi
$$

envoie $I_{X}$ sur $I_{Y}$. Il induit un automorphisme d'algèbres de Lie

$$
\Phi: \mathbb{D} \rightarrow \mathbb{D}, \quad D \mapsto \phi^{*} \circ D \circ\left(\phi^{*}\right)^{-1}
$$

qui envoie $\mathbb{D}_{Y}$ sur $\mathbb{D}_{X}$ par définition des algèbres tangentes. On obtient ainsi un isomorphisme d'algèbres de Lie

$$
\Phi: \mathbb{D}_{Y} \rightarrow \mathbb{D}_{X}
$$


Réciproquement, les théorèmes suivants étudient dans quelle mesure l'isomorphisme $\phi$ est déterminé par $\Phi$ et donc la variété par son algèbre tangente.

Théorème 3. Soient $X$ et $Y$ des sous-ensembles algébriques non vides de $\mathbb{R}^{n}$. Alors tout isomorphisme $\Phi: \mathbb{D}_{Y} \rightarrow \mathbb{D}_{X}$ d'algèbres de Lie abstraites est induit par un isomorphisme algébrique (unique) $\varphi$ de $\mathbb{R}^{n}$ qui envoie $X$ sur $Y$.

Démonstration. La démonstration est analogue à celle de $[\mathrm{H}-\mathrm{M}]$, section II.6. Tout point $P$ de $R^{n} \backslash X$ définit une sous-algèbre maximale $\mathbb{D}_{X, P}$ balancée de codimension finie $n$ (proposition 3). L'algèbre $\Phi^{-1}\left(\mathbb{D}_{X, P}\right)$ satisfait les mêmes propriétés, et donc est de la forme $\mathbb{D}_{Y, Q}$ pour un point $Q \in \mathbb{R}^{n} \backslash Y$ (proposition 3, (d) $\Rightarrow\left(\right.$ b)). On obtient ainsi une bijection $\phi: \mathbb{R}^{n} \backslash X \rightarrow \mathbb{R}^{n} \backslash Y$. Montrons que $\phi$ est une application rationnelle partout définie. Soient $\left(x_{l}\right)$ les coordonnées sur $\mathbb{R}^{n}, \phi_{l}$ les coordonnées de l'application $\phi$. Pour un champ $D \in$ $\mathbb{D}_{X}$, posons $D_{l}=\Phi\left(x_{l} \cdot \Phi^{-1}(D)\right)$. Alors

$$
\Phi^{-1}\left(D_{i}-\Phi_{i}(P) \cdot D\right)=\left(x_{i}-\phi_{l}(P)\right) \Phi^{-1}(D)
$$

est un champ de $\mathbb{D}_{Y}$ qui s'annule en $Q=\phi(P)$. Donc, si $P \in \mathbb{R}^{n} \backslash X, D_{l}$ $\phi_{i}(P) \cdot D$ est un champ de $\mathbb{D}_{X}$ qui s'annule en $P$. Posons $D=\sum b_{j} \partial_{\gamma_{j}}$ et $D_{l}=$ $\sum a_{i j} \partial_{\mathrm{r}_{j}}$ avec $b_{j}, a_{l j} \in \mathbb{R}[X]$. On déduit alors de ce qui précède que $a_{l j}(P)=$ $\phi_{l}(P) b_{J}(P)$ pour tout $P \in \mathbb{R}^{n} \backslash X$, d'où $a_{l j}=\phi_{i} b_{j}$ sur $\mathbb{R}^{n} \backslash X$. Choisissons le champ $D \in \mathbb{D}_{X}$ de la forme $D=b \partial_{\iota_{1}}$ avec $b \in I_{X}$ non nul en $P$. On alors comme ci-dessus $\phi_{l}=\frac{a_{11}}{b}$, et donc $\phi$ est régulier en $P$. En complexifiant la situation, on obtient une application régulière $\phi^{\mathbb{C}}: \mathbb{C}^{n} \backslash X^{\mathbb{C}} \rightarrow \mathbb{C}^{n} \backslash Y^{\mathbb{C}}$. Si $X$ est de codimension $\geq 2, X^{\mathbb{C}}$ l'est aussi, et le théorème d'extension de Riemann prouve que $\phi^{\mathbf{C}}$ (et donc aussi $\phi$ ) s'étend en une application partout définie. Si $X$ est une union $X_{1} \cup X_{2}$ d'une hypersurface $X_{1}$ et d'un ensemble algébrique $X_{2}$ de codimension au moins 2 , on choisit une équation $h=0$ pour $X_{1}$, et on lui associe le champ $D=b\left(\partial_{\lambda_{h}} h \partial_{\gamma_{1}}-\partial_{\lambda_{1}} h \partial_{\gamma_{h}}\right)$ avec $b \in I_{X_{2}}$ non nul en $P \in \mathbb{R}^{n} \backslash X$. Alors $D$ appartient à $D_{X}=\mathbb{D}_{X_{1} \cup X_{2}}=\mathbb{D}_{X:} \cap \mathbb{D}_{X_{2}}$ et l'argument ci-dessus montre que $\phi$ est régulière sur $\mathbb{R}^{n} \backslash\left(X_{2} \cup \operatorname{Sing} X_{1}\right)$. Il est clair que $\phi$ envoie $X$ sur $Y$ et induit l'isomorphisme $\Phi$.

On considère la situation analogue, mais pour les germes analytiques dans $\left(\mathbb{R}^{n}, 0\right)$. Mettons sur $\mathcal{O}_{n}$ la topologie analytique de convergence des coefficients. Alors $\mathbb{D}$ devient une algèbre de Lie topologique (cf. $[H-M]$, p. 329; sa topologie est définie comme module libre de rang $n$ sur $\left(\mathbb{C}_{n}\right)$, et il en est de même pour $\mathbb{D}_{X}$, si $X \subset\left(\mathbb{R}^{n}, 0\right)$ est un germe analytique.

Théorème 4. Soient $X$ et $Y$ des germes non vides de sous-variétés analytiques de $\mathbb{R}^{n}$. Supposons $n \geq 3$ et munissons $\mathbb{D}_{X}$ et $\mathbb{D}_{Y}$ de la topologie comme module de type fini sur $\mathcal{O}$ induite par la topologie analytique de $\mathcal{O}$. Alors tout 
isomorphisme topologique $\Phi: \mathbb{D}_{Y} \rightarrow \mathbb{D}_{X}$ d'algèbres de Lie abstraites topologiques est induit par un isomorphisme analytique local (unique) $\varphi$ de $\mathbb{R}^{n}$ qui envoie $X$ sur $Y$.

Démonstration. Cette démonstration, moins directe, est aussi valable pour le théorème 3. Notons $\Psi=\Phi^{\mathrm{C}}: \mathbb{D}_{Y}^{\mathrm{C}} \rightarrow \mathbb{D}_{X}^{\mathrm{C}}$ l'isomorphisme complexe $\Phi^{\mathrm{C}}=$ $\Phi \otimes \operatorname{Id}_{\mathbb{D}^{\mathrm{C}}}$ d'algèbres de Lie abstraites topologiques complexes induit par $\Phi$. Comme $\mathbb{D}_{X}^{\mathrm{C}}=\mathbb{D}_{X^{\mathrm{C}}}^{\mathrm{C}}$, le théorème II.1 de $[\mathrm{H}-\mathrm{M}]$ implique que les germes complexes $X^{\mathbf{C}}$ et $Y^{\mathbf{C}}$ sont isomorphes par un isomorphisme local $\psi$ de $\mathbf{C}^{n}$ qui induit $\Psi$. Il suffit donc de montrer que $\psi$ est le complexifié $\varphi^{\mathrm{C}}$ d'un isomorphisme local $\varphi$ de $\mathbb{R}^{n}$. Pour ceci, rappelons brièvement la construction de $\psi$ selon [H-M, II.1]. On choisit un champ $D \in I_{X} \mathbb{D}^{\mathbf{C}}$ et prouve que pour tout $f \in \mathcal{O}^{\mathbf{C}}$

$$
\Psi\left(f \cdot \Psi^{-1}(D)\right)=c_{f} \cdot D
$$

avec un élément $c_{f} \in \mathcal{O}^{\mathbf{C}}$ indépendant de $D$. Alors l'application $f \rightarrow c_{f}$ est un automorphisme de $\mathcal{O}^{\mathrm{C}}$ qui est l'application duale de $\psi$. Comme $\Psi=\Phi^{\mathrm{C}}$ il suit que pour tout $D \in I_{X} \mathbb{D}$ et tout $f \in \mathcal{O}$ réel, $c_{f} \in \mathcal{O}$ est de nouveau réel. Soit $\varphi$ l'isomorphisme local de $\mathbf{R}^{n}$ dual à $f \rightarrow c_{f}$ pour $f \in \mathcal{O}$. Alors $\psi=\varphi^{\mathbf{C}}$ et le théorème est démontré.

\section{Bibliographie}

[A-M] Atiyah, M. F. and Macdonald, I. G., Introduction to Commutative Algebra, AddisonWesley, 1969.

[H-M] Hauser, H. and Müller, G., Affine varieties and Lie algebras of vector fields, Manuscr. Math., 80 (1993), 309-337.

[H] Hironaka, H., Introduction to real analytic sets and real analytic maps, Università di Pisa, 1973.

[H2] —, Stratification and Flatness. In: Real and Complex singularities, Oslo, Sijthoff and Noordhoff, 1976.

[M] Matsumura, H., Commutative Algebra, Benjamin, 1980.

[M2] - Commutative ring theory, Cambridge, 1986.

[O] Omori, H., A method of classifying expansive singularities, J. Diff. Geom., 15 (1980), 493-512.

[R] Risler, J.-J., Le théorème des zéros en géométrie algébrique et analytique réelle, Bull. Soc. Math. Fr., 104, 2 (1976), 113-127.

[Ru] Ruiz, J. M., The basic theory of power series, Vieweg, 1993.

$[\mathrm{S}]$ Seidenberg, A., Differential ideals in rings of finitely generated type, Amer. J. Math., 89 (1967), 22-42. 
